\title{
Inductive electromagnetic data interpretation using a 3D distribution of 3D magnetic or electric dipoles
}

\author{
Michal Kolaj ${ }^{1}$ and Richard Smith ${ }^{1}$
}

\begin{abstract}
In inductive electromagnetics, the magnetic field measured in the air at any instant can be considered to be a potential field. As such, we can invert measured magnetic fields (at a fixed time or frequency) for the causative subsurface current system. These currents can be approximated with a 3D subsurface grid of 3D magnetic (closed-loop current) or electric (line current) dipoles whose location and orientation can be solved for using a potential-field-style smooth-model inversion. Because the problem is linear, both inversions can be solved quickly even for large subsurface volumes; and both can be run on a single data set for complementary information. Synthetic studies suggest that for discrete induction dominated targets, the magnetic and electric dipole inversions can be used to determine the center and top edge of the target, respectively. Furthermore, the orientation of plate
\end{abstract}

targets can be estimated from visual examination of the orientations of the 3D vector dipoles and/or using the interpreted location of the center and top edge of the target. In the first field example, ground data from a deep massive sulfide body (mineral exploration target) was inverted and the results were consistent with the conclusions drawn from the synthetic examples and with the existing interpretation of the body (shallow dipping conductor at a depth of approximately $400 \mathrm{~m}$ ). A second example over a near-surface mine tailing (a near-surface environmental/engineering study) highlighted the strength of being able to invert data using either magnetic or electric dipoles. Although both models were able to fit the data, the electric dipole model was considerably simpler and revealed a southwest-northeast-trending conductive zone. This fast approximate 3D inversion can be used as a starting point for more rigorous interpretation and/or, in some cases, as a stand-alone interpretation tool.

\section{INTRODUCTION}

The goal of most electromagnetic (EM) surveys is to produce an image of the electrical properties of the subsurface, which can explain the measured EM response. In inductive EM, there are a variety of methods available, and they range from simple and approximate back-of-the-envelope-style calculations to sophisticated and numerically intensive 3D inversions, which adhere to the full physics of the problem. Although full physics 3D inversions, such as the ones suggested by Haber et al. (2007), Cox et al. (2010), and Oldenburg et al. (2013), are increasing in popularity, their widespread use is limited due to their inherent complexity, which restricts their availability and increases their cost (monetarily and in time). As such, many prefer to use simplified approaches whereby the dominant method depends strongly on the system used, the geology, and the goal of the survey.
In airborne EM, 1D apparent conductivity imaging methods (i.e., converting amplitude and time pairs into corresponding conductivity and depth pairs) and layered earth inversions are predominant, and they are typically stitched into 2D sections or 3D volumes (Macnae and Lamontagne, 1987; Macnae et al., 1991; Smith et al., 1994; Sattel, 1998; Christensen, 2002; Huang and Rudd, 2008). Although imaging and layered earth methods are still routinely used in ground EM, user-driven iterative (i.e., trial-and-error) forward modeling using semifixed conductor shapes (i.e., parametric models) is equally if not more popular especially in certain areas, such as in mineral exploration within the Canadian Shield. The most frequently used conductor models are thin plates (West et al., 1984; Macnae and Lamontagne, 1987; Nabighian and Macnae, 1991; Liu and Asten, 1993; Smith, 2000; Kolaj and Smith, 2013, 2014), prisms (Murray et al., 1999; Sattel, 2004), and dipoles (King and Macnae, 2001; Sattel and Reid, 2006; Smith and Salem, 2007;

\footnotetext{
Manuscript received by the Editor 18 May 2016; revised manuscript received 9 January 2017; published online 25 May 2017.

${ }^{1}$ Laurentian University, Department of Earth Sciences, Sudbury, Ontario, Canada. E-mail: mkolaj@ geosci.ca; rssmith@laurentian.ca.

(C) 2017 Society of Exploration Geophysicists. All rights reserved.
} 
Schaa and Fullagar, 2010; Kolaj and Smith, 2015). The forward operator in these parametric models is considerably less complicated than that in 3D models that describe the full physics, and, as such, they can often be incorporated into automated inversion routines. This is especially true for the dipole model, and, as such, there are many examples of semiautomated to fully automated inversion routines using dipoles. For example, Smith and Salem (2007) and Kolaj and Smith (2015) use free-space magnetic dipole look-up tables to fit airborne and ground EM data. Sattel and Reid (2006) use a combination of magnetic and electric dipoles (crossstrike directed line current) embedded in a layered earth to fit spatially discrete airborne EM anomalies. There is also considerable research into dipole-based interpretation within the unexploded ordinance community (Pasion and Oldenburg, 2001; Beran et al., 2013). Although the work described above generally fits discrete EM anomalies with single dipoles, using the concepts of moments (Smith and Lee, 2001, 2002), Schaa and Fullagar (2010) and Fullagar et al. (2015) develop a 3D inversion that fits resistive-limit EM data using a discretized subsurface grid of magnetic dipoles. By using resistive-limit data, they are able to take full advantage of potential-field-style linear inversion, which is significantly faster than traditional 3D EM inversion. Because dipole-based inversion can provide significant information at a low cost, it is an attractive choice, especially for preliminary, short-turnaround interpretations.

Under the quasistatic assumption (i.e., negligible displacement current), the magnetic field $(\mathbf{H})$ vector wave equation reduces to the vector diffusion equation (Grant and West, 1965):

$$
\nabla^{2} \mathbf{H}=\sigma \mu \frac{\partial \mathbf{H}}{\partial t},
$$

which, in the air (where $\sigma=0$ ), further reduces to the vector Laplace's equation:

$$
\nabla^{2} \mathbf{H}=0 .
$$

By dropping the time-dependent term from equation 1, equation 2 implies that the magnetic field in the air is not influenced by its past history. As such, it is a potential field and, at any point in time, it can be determined exactly from the subsurface current distribution at that time (Nabighian and Macnae, 1991). In our work, we assume that at a given fixed time, that subsurface current distribution can be approximated with a 3D subsurface grid of static magnetic (a unit area circular current loop) or electric (a small current element) dipoles. As such, we can use the measured secondary magnetic field at a fixed time or frequency to quickly solve for a 3D distribution of subsurface dipoles using a potential-field style inversion similar to Schaa and Fullagar (2010). By using the resistive limit, Schaa and Fullagar (2010) can effectively only determine one current distribution, but our approach can determine the amplitude and orientation of the dipoles (which can be either magnetic or electric) at a single time or for a series of times, and can therefore provide significant detail about the location and migration of currents in the subsurface. This knowledge can be used as is or as a starting model for more rigorous interpretation.

We begin by presenting our forward and inversion methodology, which we test on a synthetic plate target example. The inversion is then tested on two fixed-loop ground surveys. The first example consists of a single receiver component survey over a deep massive sulfide body (mineral exploration example), whereas the second example uses $3 \mathrm{C}$ receiver data collected over a near-surface tailing pond, which was the focus of an environmental and engineering study.

\section{METHODOLOGY}

In the forward model, the magnetic field at the measurement station is calculated from the sum of the magnetic fields generated by a discretized subsurface grid of 3D cells with three orthonormal dipoles (dipoles oriented along the $x$-, $y$-, and $z$-axes) in each cell center. This can be written mathematically for $n$ total cells as

$$
\mathbf{H}^{\mathrm{S}}(\mathbf{s})=\sum_{k=1}^{n} \mathbf{M}_{k} \mathbf{G}_{k}(\mathbf{s}) V_{k},
$$

where $\mathbf{s}$ corresponds to the position vector of the station location, the vector $\mathbf{M}_{k}$ corresponds to the moment of each dipole (units of $\mathrm{Am}^{2}$ and $\mathrm{Am}$ for magnetic and electric dipoles, respectively) within cell $k$, and $\mathbf{G}_{k}$ is a tensor corresponding to the nine components of magnetic fields generated by the three dipoles centered within cell $k$ of volume $V_{k}$ (dimensionless scalar). In our formulation, $\mathbf{G}$ is constructed from three separate vectors $\left(\mathbf{g}_{i}\right)$, which for a magnetic dipole is (Ward and Hohmann, 1988)

$$
\mathbf{g}_{i}(\mathbf{s})=\frac{1}{4 \pi|\mathbf{r}-\mathbf{s}|^{3}}\left[\frac{3 \hat{\mathbf{m}}_{i} \cdot(\mathbf{r}-\mathbf{s})}{|\mathbf{r}-\mathbf{s}|^{2}}(\mathbf{r}-\mathbf{s})-\hat{\mathbf{m}}_{i}\right],
$$

and for an electric dipole is (Ward and Hohmann, 1988)

$$
\mathbf{g}_{i}(\mathbf{s})=\frac{\hat{\mathbf{m}}_{i} \times(\mathbf{r}-\mathbf{s})}{4 \pi|\mathbf{r}-\mathbf{s}|^{3}}
$$

where $\mathbf{r}$ is the position vector of the cell center, $\hat{\mathbf{m}}$ is equal to the unit vector of the dipole in each of the three cardinal directions, so that $i$ refers to the directional axis of the dipole (either $x, y$, or $z$ ). The forward model (equations 3-5) solves for the magnetic field produced by a subsurface distribution of orthogonal magnetic or electric dipoles, and, in this work, we use the moment of those dipoles (M) as a proxy to the established current system.

In the inverse problem, we are attempting to solve for an equivalent distribution of dipoles that match the measured magnetic field at a particular instance in time (or at a specific frequency). The forward model does not explicitly take any background medium into consideration, so the inverse problem is applicable for discrete targets embedded in a resistive half-space. If a background response is present, a possible option would be to strip the background response (Smith and Salem, 2007) and/or use a late-enough delay time (or a low-enough frequency), in which the background response is small. Alternatively, our formulation could also be used to find a subsurface current distribution that explains the background response of the conductive host in addition to the anomalous response. Because there is no temporal variable in equations $3-5$, the problem must be solved separately for each frequency or time. Solving the system at multiple frequencies or at multiple times could provide the method with additional sensitivity to the conductivity of the target(s).

The system is typically overdetermined and can be solved by minimizing (two-norm) the functional $f(\mathbf{M})$

$$
\begin{aligned}
f(\mathbf{M})= & \left\|\mathbf{W}_{\sigma}\left(\mathbf{G M}-\mathbf{H}^{\mathrm{S}}\right)\right\|^{2}+\alpha_{x}\left\|\mathbf{W}_{x} \mathbf{Z M}\right\|^{2}+\alpha_{y}\left\|\mathbf{W}_{y} \mathbf{Z M}\right\|^{2} \\
& +\alpha_{z}\left\|\mathbf{W}_{z} \mathbf{Z M}\right\|^{2}+\alpha_{s}\left\|\mathbf{W}_{s} \mathbf{Z M}\right\|^{2},
\end{aligned}
$$

where $\mathbf{M}$ is the matrix of dipole moments that are being solved for, $\mathbf{G}$ is the matrix representation of the forward model operator from 
equations 3 to 5 (with inclusion of the volume term $V$ ), and $\mathbf{H}^{\mathrm{S}}$ are the measured magnetic fields to be fit. The weighting matrices $\mathbf{W}_{x}$, $\mathbf{W}_{y}$, and $\mathbf{W}_{z}$ are the smoothing regularization matrices (the first finite-difference operators) that smooth each dipole moment in each of the three Cartesian directions, $\mathbf{W}_{\sigma}$ is a weighting matrix corresponding to the inverse of the data error (if known), and $\mathbf{W}_{s}$ encourages model smallness (i.e., minimum complexity). Depth weighting is applied with the diagonal matrix $\mathbf{Z}$, which, like in potential-field inversion, is necessary so as to counteract the rapid drop off in amplitude of the magnetic field with distance (equations 4 and 5). Without adequate depth weighting, the solution will favor a nearsurface model (i.e., the dipoles are concentrated in the top layer(s) of the discretized subsurface) regardless of the true depth of the causative features. To solve this issue, we adopt the depth-weighting scheme from Li and Oldenburg (1996)

$$
Z_{i i}=z^{-\beta / 2}
$$

where the values of $Z_{i i}$ make up the entries of the diagonal matrix $\mathbf{Z}$ and $z$ is the depth from the average station elevation to the center of the subsurface cell. A natural choice for $\beta$ would be the fall-off rate of the dipole amplitude (i.e., $\beta=3$ for magnetic dipoles and $\beta=2$ for electric dipoles), but in our experience, leaving $\beta=3$ in almost all circumstances produced favorable results. Equation 3 is typically underdetermined, and there exists more than one model that will exactly fit the data. However, by minimizing equation 6 , we impose additional constraints by solving for a model with specified regularized properties. The regularization parameters $\left(\alpha_{x}, \alpha_{y}, \alpha_{z}\right.$, and $\left.\alpha_{s}\right)$ control the relative influence of the smoothing matrices and the model smallness (and together the influence of depth weighting) as compared with the data misfit (the first term in equation 6). Because equation 6 (the inverse problem) can be solved in a few seconds using a conjugategradient method implemented in MATLAB, it is possible to solve it for many different regularization parameters, and thus, models. In our implementation, we generally solve for the optimum $\alpha$ values using a combination of an L-curve analysis (Zhdanov, 2002) and a qualitative analysis of the solutions obtained. In this manner, we aim to select a representative model from the set of solutions, which is a balance between minimization of the model and data norms.

It should be noted that the inversion (equations 4-6) solves for three orthogonal dipole moments within each cell (i.e., a vectordipole moment) and each dipole moment direction $(x, y$, and $z$ ) can be analyzed/interpreted separately. However, for imaging and interpretation purposes, it is preferable to convert the vector-dipole moment into a scalar value by taking the magnitude of the dipole moment vector within each cell, and we represent this value as $\left|\mathbf{M}_{m}\right|$ or $\left|\mathbf{M}_{e}\right|$ (the magnitude of the magnetic or electric vector dipoles, respectively). As we lose the orientation information by using a scalar magnitude, we also plot the vector-dipole moments using vector fields (generally only those with a magnitude above a certain threshold). In this manner, we use the magnitude as a proxy to the strength of the established current system and the vector fields as an indication of the orientation of that current system.

\section{SYNTHETIC EXAMPLE}

Two time-domain fixed-loop ground surveys using $400 \times 200 \mathrm{~m}$ $50 \mathrm{~S}$ (conductance) plates embedded in a resistive half-space were simulated in GeoTutor (PetRos EiKon) using the VHPlate algorithm (Walker and West, 1991), and the survey geometry (line and station spacing was 150 and $50 \mathrm{~m}$, respectively), plate properties, and $z$-component response from the central line are shown in Figure 1. For our inverse problem, the subsurface was discretized into $25 \times 25 \times 25 \mathrm{~m}$ cells (easting, northing, and depth, respectively) and the inversion (equation 6) was run for a late off-time channel ( $t=9.4 \mathrm{~ms} ; 30 \mathrm{~Hz}$ base frequency) for magnetic and electric dipoles. The computation times for the inversions presented were all generally less than $10 \mathrm{~s}$ (per suite of regularization parameters) and the root-mean-square error for all inversions was less than $10^{-2}$.

In the first example, a $140^{\circ} / 30^{\circ}$ southwest (strike/dip) plate (plate 1, Figure 1) with a depth to the top of $250 \mathrm{~m}$ was used and the inversion results $\left(\left|\mathbf{M}_{m}\right|\right.$ and $\left.\left|\mathbf{M}_{e}\right|\right)$ for the magnetic and electric dipoles are shown in Figure 2. For the magnetic dipole inversion, the dipoles were concentrated around the center of the plate with the largest amplitude dipole being located slightly southwest (in the dip
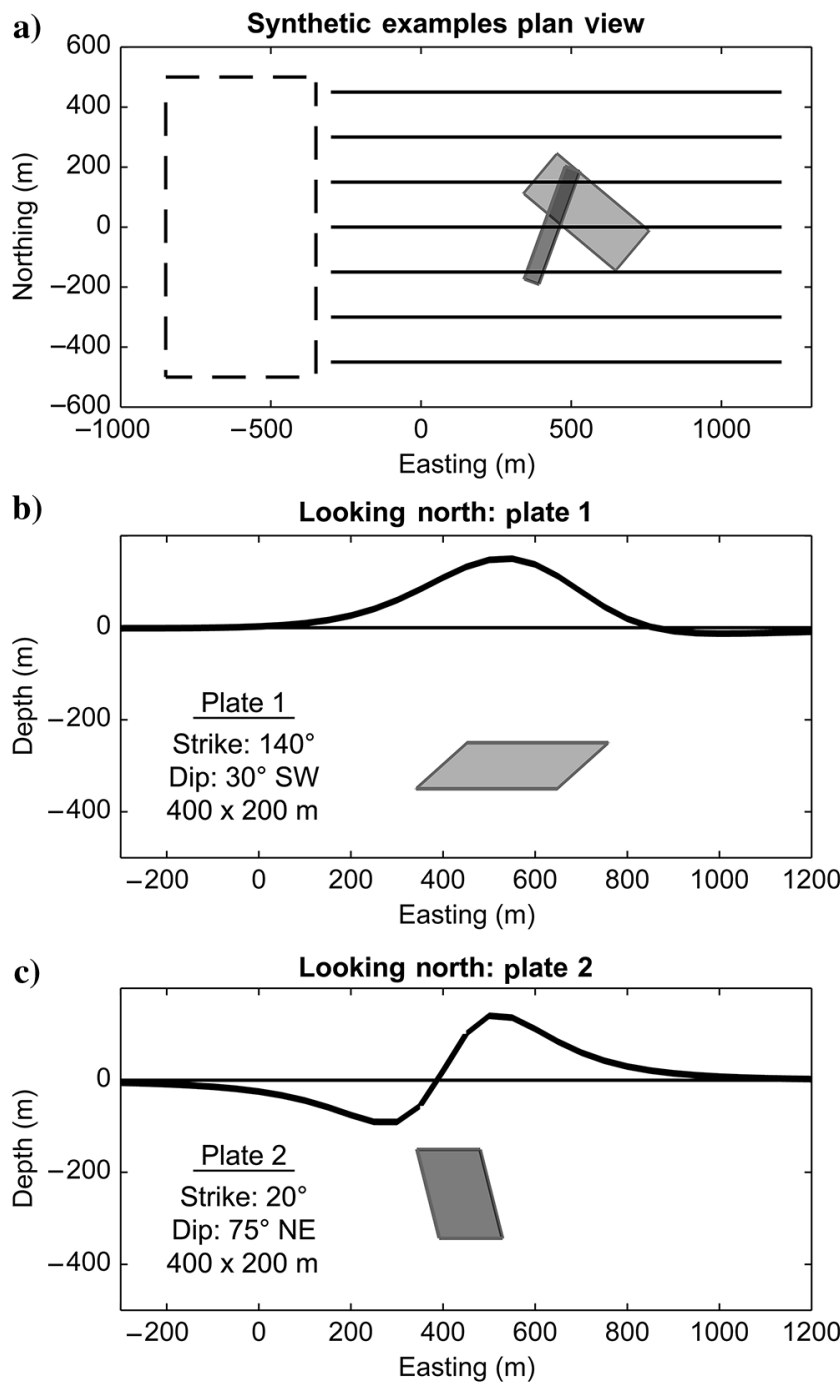

Figure 1. (a) Plan view of the survey geometry of the two synthetic models (plates 1 and 2) simulated in GeoTutor. Transmitter loop is shown with a dashed line, and the station lines are depicted with thin solid black lines. (b) First synthetic example (plate 1, gray plate) consisted of a $140^{\circ} / 30^{\circ}$ southwest (strike/dip) plate (plate 1) with a depth to the top of $250 \mathrm{~m}$. (c) Second synthetic example (plate 2 , black plate) consisted of a $20^{\circ} / 75^{\circ}$ northeast plate (plate 2) with a depth to the top of $150 \mathrm{~m}$. The $z$-component response $(t=9.4 \mathrm{~ms})$ for the central line for both surveys is shown with a thick black line. 
direction) of the plate center. Although the location matches well with the actual location of the plate, the general shape of the anomalous zone does match the strike or dip of the plate. This information is better resolved with the electric dipole inversion, which fit the dipoles along the top edge of the plate (peak dipole at a depth of $-225 \mathrm{~m}$ ) with the anomalous zone oriented parallel with the true strike of the plate. Because the magnetic inversion indicated the center of the plate and the electric inversion indicated the top edge, it is possible to estimate the dip of the target, which in this case is calculated to be $31^{\circ}$, which matches the true dip of $30^{\circ}$.

In the second example, a $20^{\circ} / 75^{\circ}$ northeast (strike/dip) plate (plate 2, Figure 1) with a depth to the top of $150 \mathrm{~m}$ was used and the inversion results $\left(\left|\mathbf{M}_{m}\right|\right.$ and $\left.\left|\mathbf{M}_{e}\right|\right)$ for the magnetic and electric dipoles are shown in Figure 3. As with the previous example, the magnetic dipoles were concentrated around the center of the plate and the largest amplitude dipole is located slightly away (in the dip direction) from the true plate center. However, unlike the previous example, the strike and dip direction is roughly reflected in the shape of the magnetic dipole anomaly, whereby there is a "tail" of anomalous dipoles that extends away from the plate opposite to the dip direction. This tail was observed in other synthetic examples, especially when the plate was steeply dipping. As before, the electric dipole inversion clusters parallel to the top edge of the plate (peak dipole at a depth of $-125 \mathrm{~m}$ ) and the strike direction can be clearly inferred. Calculating the dip using the location of the peak electric and magnetic dipoles suggests a dip of $60^{\circ}$, which is smaller
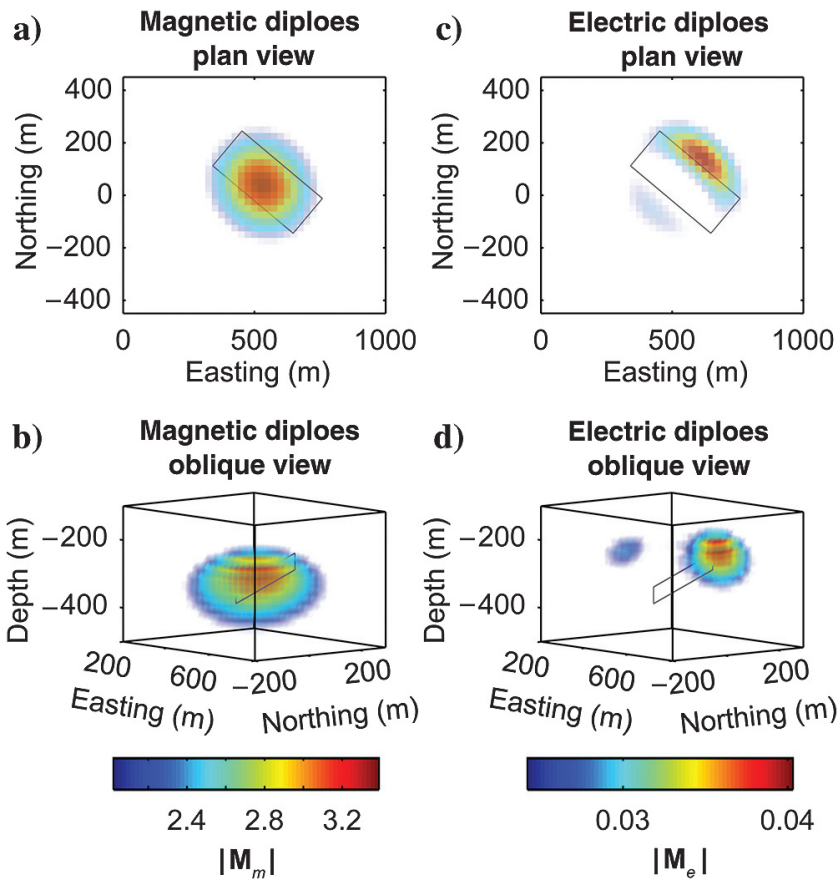

Figure 2. (a, c) Plan and (b, d) oblique view of the results of the $(a, b)$ magnetic and (c, d) electric dipole inversions (equations 2 and 4; the regularization parameters $\alpha_{x}, \alpha_{y}, \alpha_{z}$, and $\alpha_{s}$ were equal to 0.007 and 0.03 for the magnetic and electric dipole inversions, respectively) for survey 1 (plate 1, Figure 1). The magnitude of the vector-dipole moment $\left(\left|\mathbf{M}_{m}\right|\right.$ and $\left.\left|\mathbf{M}_{e}\right|\right)$ at each location is depicted, whereby hotter colors represent higher amplitude dipoles. Magnetic and electric dipoles with magnitudes less than $2 \mathrm{Am}^{2}$ and $0.025 \mathrm{Am}$, respectively, are not shown. The outline of the plate target is shown with the darkgray line. than the true dip of $75^{\circ}$. This discrepancy is likely due to the fact that the peak electric dipole is slightly above the true location of the plate and that the peak magnetic dipole is located away from the true center of the plate. The error these discrepancies introduce into the dip calculation is also predicted to increase with the increasing dip of the target.

As was mentioned in the "Methodology" section, the general orientation of the current system features can also be determined by examination of the vector orientation of the dipoles. For plate-like targets, the directionality of the current system will coincide with the orientation of the plate (i.e., indifferent to the source-field direction). A vector-field map for both synthetic examples is plotted in Figure 4. In the top panel of Figure 4, the electric dipole vector orientations are shown in a plan view. The electric dipoles define a horizontal current system whose direction is parallel with the strike of the target where, again, the largest amplitude dipoles are located roughly along the top edge of the plate target. In the bottom panel of Figure 4, the magnetic dipole vector orientations are shown in the exact oblique view as in Figures 2 and 3. In all cases, the peak amplitude magnetic dipole underestimates the true dip of the plate, but the magnetic dipoles coincident with the actual location of the plate accurately reflect the true dip of the plate (normal to the plate). Without prior knowledge, it may be difficult to ascertain an exact estimate of the dip because the true dip is not reflected in the largest amplitude dipole. However, in our experience, an examination (visual or quantitatively using a statistical approach) of the general orientation of the vector fields (strike from electric dipoles, and dip
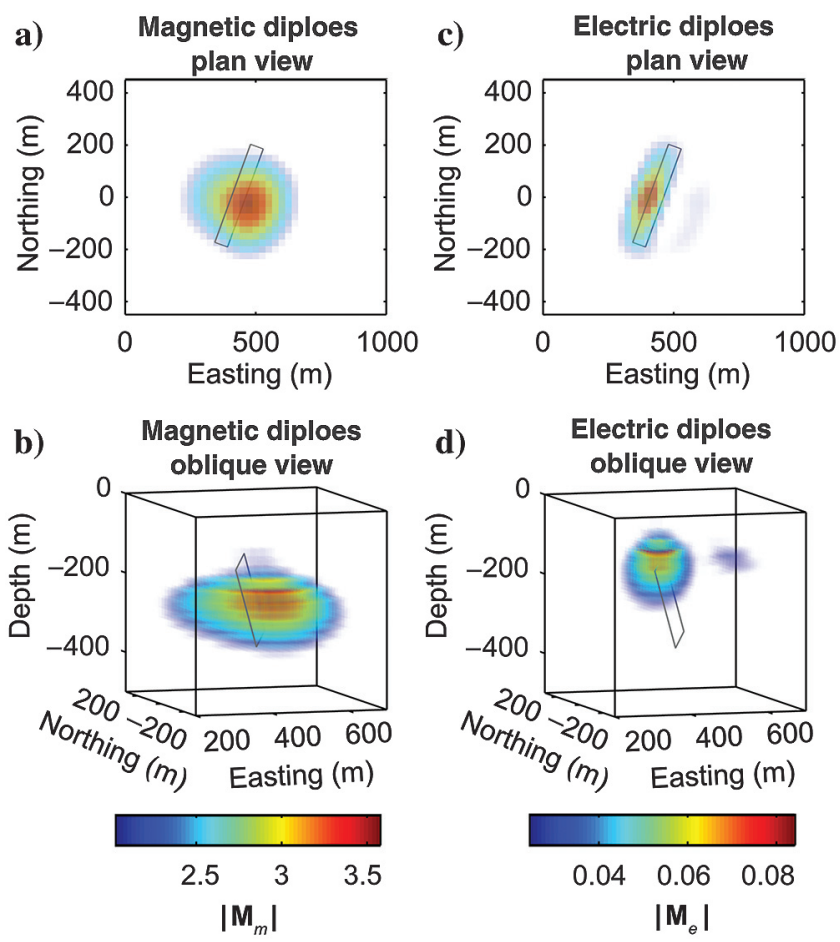

Figure 3. (a, c) Plan and (b, d) oblique views of the results of the $(a, b)$ magnetic and (c, d) electric dipole inversions (equations 2 and 4; the regularization parameters $\alpha_{x}, \alpha_{y}, \alpha_{z}$, and $\alpha_{s}$ were equal to 0.006 and 0.009 for the magnetic and electric dipole inversions, respectively) for survey 2 (plate 2, Figure 1). Magnetic and electric dipoles with magnitudes less than $2 \mathrm{Am}^{2}$ and $0.025 \mathrm{Am}$, respectively, are not shown. The outline of the plate target is shown with the dark-gray line. 
from magnetic dipoles) provides a reliable estimate of the general orientation of the target. Moreover, by using all available information (the magnitude, dip estimate from magnetic to electric dipole centers, and the vector fields), it is possible to accurately and quickly estimate the location and orientation of plate targets. As these inversions are fast and simple to use, they do not require an initial guess and can be run as a preliminary step to gain insight into the subsurface geology. Moreover, the results could be used to guide a starting model for the more time-consuming interpretation routines, such as iterative forward modeling or inverse modeling that requires an initial guess.

\section{FIELD EXAMPLES}

In the following section, we present two field examples of the dipole inversions. In the first example, the inversion is run on a deep mineral exploration target, whereas the second example is concerned with the characterization of a near-surface tailings pond surveyed for environmental and engineering applications. The success of the inversion on these two very different examples aims to showcase the generality and potential applications of this method.

\section{Deep mineral exploration}

The Joe Lake property is located in the north range of the Sudbury Igneous Complex, and it contains a deep, shallow-dipping sulfide body, which was discovered with a ground EM UTEM (West et al., 1984) survey (Watts, 1997). The example showcases the ability of ground EM to discover deep conductive targets as the late time-channel data showed a distinct anomaly over four to five lines. The ground EM survey consisted of single vertical component $\left(B_{z}\right)$ data at a nominal station and line spacing of $50 \times 100 \mathrm{~m}$, respectively $(1.9 \times 2.5 \mathrm{~km}$ transmitter loop with the closest edge being approximately $900 \mathrm{~m}$ east of the delineated target).
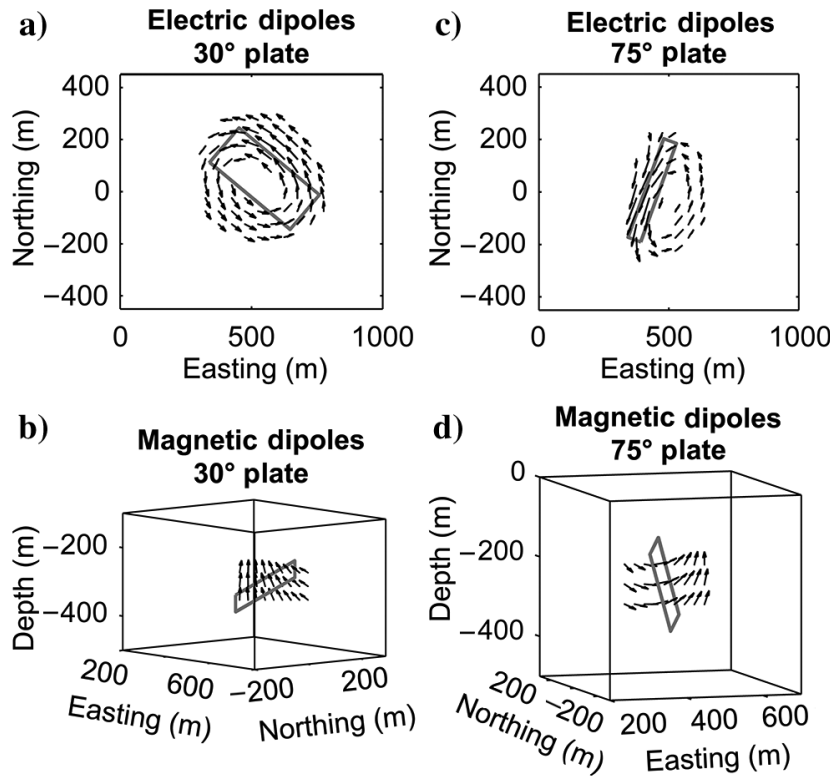

Figure 4. (a, c) Plan and (b, d) oblique view of the dipole moment vectors corresponding to the magnetic and electric dipole inversion models for both synthetic examples from Figures 2 and 3. The outline of the plate target is shown with the dark-gray line.
For the dipole inversion, the subsurface was discretized into $25 \times 25 \times 25 \mathrm{~m}$ cells (easting, northing, and depth, respectively) up to a depth of $800 \mathrm{~m}$ as well as the results and the corresponding data fit ( $t=0.7812 \mathrm{~ms}, 31 \mathrm{~Hz}$ base frequency) for four lines are shown in Figures 5 and 6 , respectively. It should be noted that
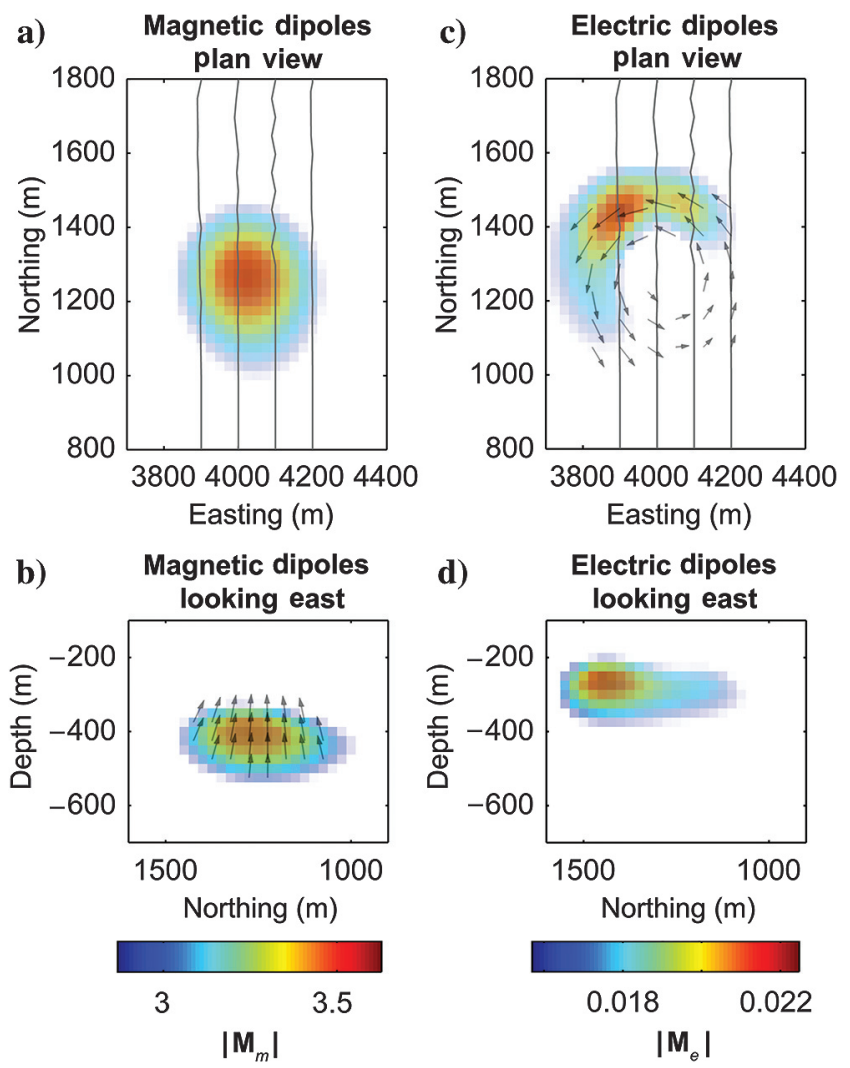

d)

Easting $(\mathrm{m})$

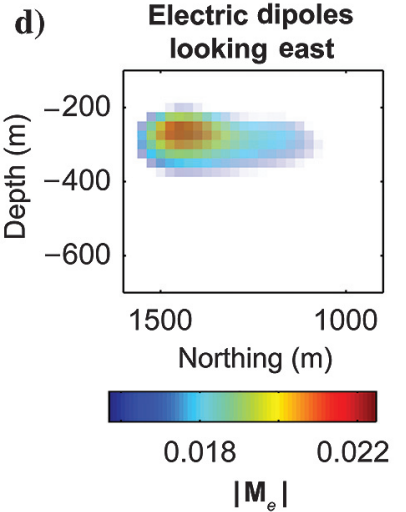

Figure 5. (a, c) Plan and (b, d) looking east view of the results of the $(\mathrm{a}, \mathrm{b})$ magnetic and (c, d) electric dipole inversions (equations 2 and 4 ; the regularization parameters $\alpha_{x}, \alpha_{y}, \alpha_{z}$, and $\alpha_{s}$ were equal to 0.008 and 0.08 for the magnetic and electric dipole inversions, respectively) for the Joe Lake survey (computation times in the order of a few seconds per inversion). Magnetic and electric dipoles with magnitudes less than $2.9 \mathrm{Am}^{2}$ and $0.016 \mathrm{Am}$, respectively, are not shown. Station lines are depicted with thin solid gray lines. Select dipole moment vectors corresponding to (c) depth $=-275 \mathrm{~m}$ and (b) easting $=4025 \mathrm{~m}$ are shown.

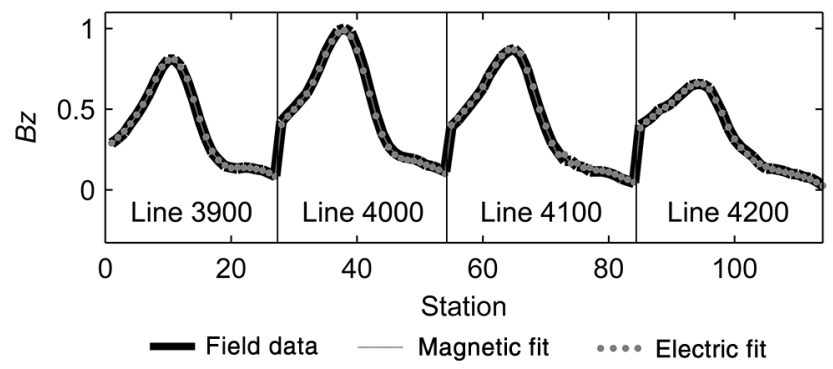

Figure 6. Comparison of the field data (thick solid black line) and the model data from the magnetic (solid gray line, Figure $5 \mathrm{a}$ and $5 \mathrm{~b}$ ) and electric (dashed gray line, Figure $5 \mathrm{c}$ and $5 \mathrm{~d}$ ) dipole inversions for the vertical component of the magnetic field. The data were normalized to the peak value. All four lines of data are shown in series (separated by solid black lines), whereby the station number increases from west to east and south to north. 
the field data were lightly smoothed with a three-point averaging filter to smooth the data at the ends of the lines, where the signalto-noise ratio was the poorest. The magnetic dipole inversion revealed a body centered at 4025, 1275, and $-400 \mathrm{~m}$ (Figure 5a and $5 \mathrm{~b}$ ), and interpretation of the vector orientations of the dipole moments (Figure 5b) suggests that the body is shallow dipping to the southeast. The electric dipole solution (Figure $5 \mathrm{c}$ and $5 \mathrm{~d}$ ) is consistent with a southeast-dipping body because the peak electric dipole $(3900,1450$, and $-275 \mathrm{~m})$ is northwest of the magnetic anomaly, and the orientation of the electric dipole moment vectors (Figure 5c) suggests a northeast-southwest-striking body. The strike and dip were calculated to be $55^{\circ}$ and $30^{\circ}$ southeast, respectively, using the peak magnetic and electric dipole locations, which also agrees with the previous interpretations.

The ground EM survey data were previously modeled and interpreted using the plate modeling software MultiLoop (Lamontagne Geophysics). They were modeled with a south-dipping $\left(30^{\circ}\right)$ plate centered at $1300 \mathrm{~N}$ with a depth to top ranging from 375 to $425 \mathrm{~m}$ (Watts, 1997), which is consistent with the results of the magnetic and electric dipole inversions. There is a slight discrepancy between the predicted depth to the top edge of the plate ( $-275 \mathrm{~m}$ from the electric dipole inversion), but in the synthetic studies, it was found that the peak electric dipole tended to be variably above the true location of the plate, which may explain the difference.
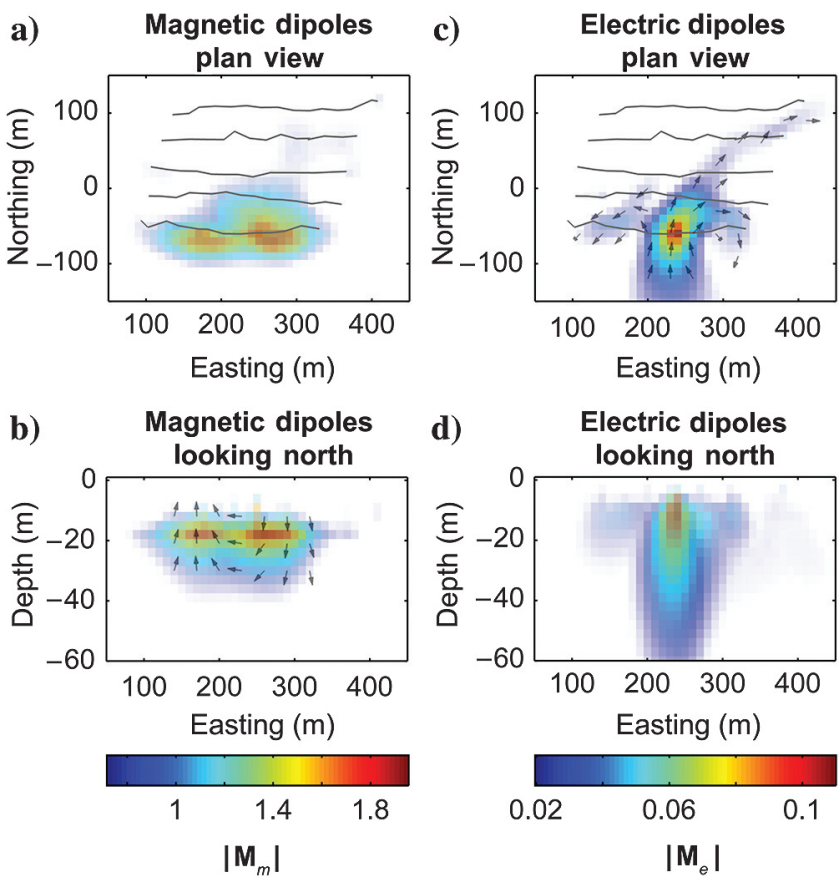

Figure 7. (a, c) Plan and (b, d) looking east view of the results of $(a, b)$ the magnetic and (c, d) electric dipole inversions (equations 2 and 4; the regularization parameters $\alpha_{x}, \alpha_{y}, \alpha_{z}$, and $\alpha_{s}$ were equal to $2 \times 10^{-3}, 2 \times 10^{-3}, 6 \times 10^{-3}, 1 \times 10^{-4}$, and $1 \times 10^{-1}, 1 \times 10^{-1}$, $6 \times 10^{-3}$, and $2 \times 10^{-2}$ for the magnetic and electric dipole inversions, respectively) for the tailing survey. Magnetic and electric dipoles with magnitudes less than $0.7 \mathrm{Am}^{2}$ and $0.02 \mathrm{Am}$, respectively, are not shown. Station lines are depicted with thin solid gray lines. Select dipole moment vectors corresponding to (c) depth $=-15 \mathrm{~m}$ and (b) northing $=-60 \mathrm{~m}$ are shown.

\section{Near-surface environmental characterization}

The second field example consists of a $3 \mathrm{C}$ fixed in-loop survey collected overtop an old dry tailings pond in Sudbury, Ontario, Canada. Tailings are the waste material produced after processing ore to extract valuable metals and can be as large as several kilometers in length and several tens of meters in height. The original survey was carried out in an effort to map the electrical properties, which could be used as a proxy to map potential contaminants, fluids, and/or anomalous concentrations of leftover metals (Kolaj and Smith, 2013, 2014). The survey consisted of five lines with a station and line spacing of approximately 20 and $40 \mathrm{~m}$, respectively, inside of a $700 \times 350 \mathrm{~m}$ transmitter loop.

The subsurface was discretized into $10 \times 10 \times 3 \mathrm{~m}$ cells (easting, northing, and depth, respectively) up to a depth of $120 \mathrm{~m}$. An early off-time channel was fit ( $t=0.295 \mathrm{~ms}, 30 \mathrm{~Hz}$ base frequency), and the results of the magnetic and electric dipole inversions and the corresponding data fit are shown in Figures 7 and 8, respectively. It should be noted that for this example, the depth-weighting matrix ( $\mathbf{Z}$ in equation 6) was removed from the smoothing operators because without this change, it was found that the inversion was unable to successfully fit a smooth near-surface model to the data.

For the magnetic dipole inversion (Figure $7 \mathrm{a}$ and $7 \mathrm{~b}$ ), most of the response could be explained via two shallow anomalies located to the south of line $50 \mathrm{~S}$. The vector dipole moments (Figure $7 \mathrm{~b}$ ) reveal that the two anomalies represent peak positive and negative $z$ directed dipole moments, which appear to be circulating around a north-south trend located at 220 east. A potential explanation
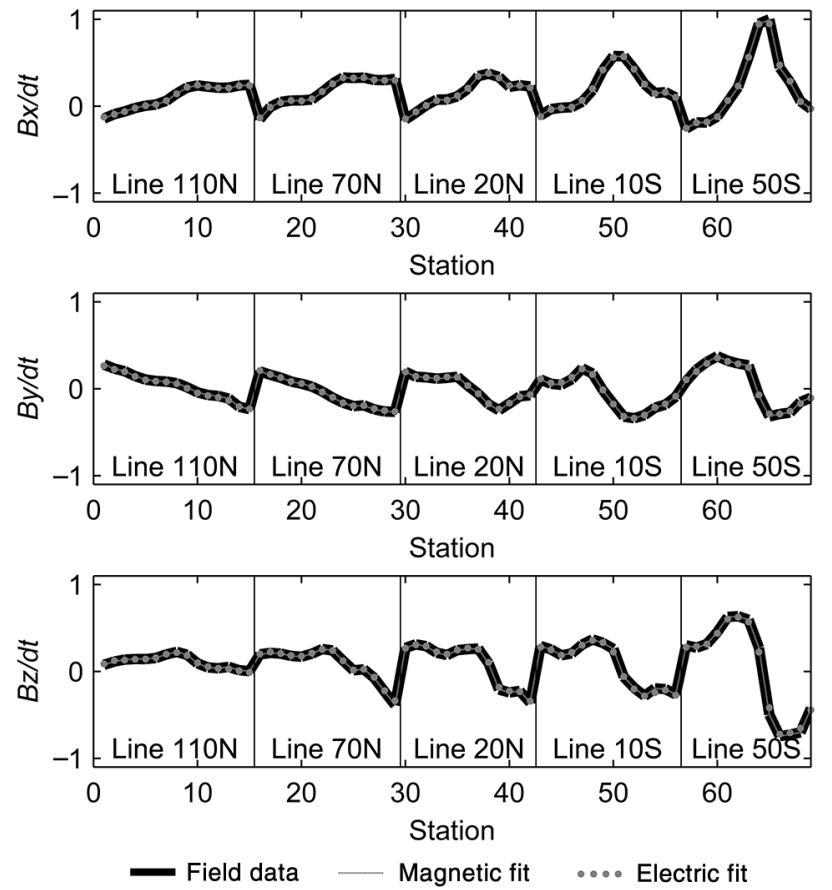

Figure 8. Comparison of the field data (thick, solid black line) and the model data from the magnetic (solid gray line, Figure $7 \mathrm{a}$ and $7 \mathrm{~b}$ ) and electric (dashed gray line, Figure 7c and 7d) dipole inversions for the $3 \mathrm{C}$ of the magnetic field. The magnetic field components were normalized to the peak value. All five lines of data are shown in series (separated by solid black lines), whereby the station number increases from west to east and north to south. 
is that the data cannot be fit with discrete magnetic dipoles and to produce the dominant $B_{z}$ crossover type response (i.e., the $z$ response in line $50 \mathrm{~S}$ ), the inversion mapped the distribution of the subsurface magnetic fields rather than an underlying causative feature. It is also possible that this is due to over-regularization, but experimentation with coarser grids and smaller smoothness constraints did not remove this feature. On the other hand, the electric dipole inversion (Figure 7c and 7d) produced a more realistic solution: a shallow north to northeast directed line current. This can also explain the circulating magnetic dipoles as magnetic fields curl around a line current (i.e., Ampere's law). There are some "curling" effects to the west and east of the peak electric dipole, and this is likely an artifact due to the smoothing regularization and/or the necessity to also include a minor magnetic dipole component. Another potential interpretation complication is mutual coupling between multiple targets and/or nonsimple conductor and/or the interactions with a nonfreespace background medium. We believe that this is unlikely due to the presence of a relatively small $B_{z}$ crossover-type anomaly centered on the southernmost line (i.e., no strong background response evident) and the good fit obtained by a rather small discrete electric dipole distribution. However, the underlying cause of the line current is unknown, but it could potentially include any conductive feature, such as a buried pipe, conductive channel of fluids/material, and/or a near-vertical feature, such as a conductive fault.

\section{DISCUSSION}

The synthetic and field examples show that typical plate discrete target responses can be reliably fit with a 3D volume of dipoles. In our experience, the near center of the plate can be determined using magnetic dipoles. Using the magnetic vector-dipole moments, the orientation corresponding to the peak dipole moment tends to underestimate the dip and a more accurate estimate can be made by an analysis of the adjacent vector dipole moments. However, this can be somewhat subjective and without prior knowledge of the true target location, it is difficult to determine which vector dipole moments are the most reliable. For induction-dominated targets, the electric dipole inversion places the dipoles at positions and orientations that are consistent with the strike of the target and tend to be in close proximity to the top of the shallowest edge of the target. Because magnetic dipoles tend to concentrate at the center of the target and the electric dipoles tend to concentrate along the top edge, the orientation of the target can be estimated using the vector that defines the peak magnetic-to-electric dipole locations. This method has been found to be effective, especially for shallow-dipping targets. Electric dipoles should be more applicable with highly elongated targets (which appears to be the case in the tailing field example) and when current channeling is the primary response (not tested in this work). Because both dipole inversions can be solved quickly even for large subsurface volumes, both can be performed for complementary information. For example, the magneticdipole inversion could be used to determine the center of the plate and the electric dipole inversion for the top edge, as was predominantly done in this work.

The methodology presented in this work relies on, first, that the inverted distribution of dipoles is a suitable proxy to the established current system and second, that it can be interpreted to discern information about the geoelectric structure of the subsurface. For the first point, a volume distribution of magnetic dipoles can reproduce an arbitrary realizable current, assuming that a fine enough discretization is used to accurately model the shape of the current system because a magnetic dipole is an infinitesimal current loop. Although the magnetic dipole representation maps the equivalent representation of the actual current system (e.g., a square line current can be modeled with a rectangular 2D distribution of magnetic dipoles), the electric dipole formulation maps the current system directly. However, it should be noted that although the smoothing regularization in the inversion encourages the electric dipole solutions to form closed-current systems (see Figures 4 and 5), the inversion does not force zero divergence (unlike the magnetic dipoles that are divergence free by definition), which implies that the current system that is solved for may not be physically realizable. An attempt to apply a soft constraint on the divergence by adding a finite-difference approximation of the divergence operator as an additional regularization matrix did substantially lower the divergence of the model, but the results were inconsistent and contained significant artifacts. Overall, because the intent of the inversion is to provide fast approximate results to guide further interpretation, the lack of this constraint was not found to be significantly detrimental.

For the second point, the interpretation of the equivalent current system represented by the magnetic and electric dipoles depends on the geologic regime being considered. In this work, we have focused on the interpretation of discrete plate targets embedded in a fully resistive medium. In this way, the interpretation of the current system is straightforward because it directly delineates the features of interest and the orientations of the vector fields are related to the orientation of the targets. In the case of nonthin-sheetlike targets, the orientation of the dipoles will be influenced by the coupling angle between the source and the target. In these cases, the orientation of the dipoles (i.e., the current system) not only reflects the orientation of the target, but it is also affected by the location and geometry of the source. Although a misinterpretation of the orientation is possible, the distribution of dipoles should still coincide with the location of the established current system. In the simple case of a fixed transmitter, this issue can be somewhat resolved by adding an additional primary field coupling term to equations 4 and 5. This method in effect applies weights between the three possible dipole directions within each cell to account for the coupling angle with the primary field. In the examples presented in this work, a preliminary attempt at applying these weights was found to have an overall detrimental effect on the interpretability of the resultant data and was not further investigated. It is suspected that this is due to the data presented in this work being well-approximated as thinsheet targets, in which the directionality of the induced current system is determined by the orientation of the target and not the primary field.

A background geologic response was also not included in our forward modeling operator or considered in our synthetic modeling because it was not found to be necessary for our area of study (resistive Canadian Shield geology), and the methodology in its current state has only been tested on relatively discrete targets in which the background response is negligible (i.e., resistive medium, latetime channel, stripped responses, etc.). A possible solution would be to incorporate a background half-space or layered earth forward model in addition to the 3D-dipole response (Sattel and Reid, 2006; Schaa and Fullagar, 2010), which would increase the generality of the methodology at the cost of increased complexity in the forward operator. Alternatively, this may be unnecessary because the electric 
and/or magnetic dipoles may be able to reliably fit a background response in addition to the anomalous response. For example, it should be possible to model the response of a conductive half-space using our formulation of electric dipoles because it can also be modeled with a closed current loop (with an identical shape to the transmitter loop), which deepens and increases in horizontal dimensions with time (Nabighian, 1979; Nabighian and Macnae, 1991). By not attempting to include a background response in the forward operator, the resultant dipole distribution attempts to map the true location of current. Similarly, we also do not consider any mutual coupling between the dipoles because we are attempting to map the strength (and direction) of the current system and not the conductivity of each cell. This has the negative effect of making the amplitudes of the dipoles more difficult to interpret (i.e., related to conductivity) because the amplitudes are not only a function of the conductivity of each cell, but also of the primary field coupling and any nonlinear effects due to the mutual coupling between dipoles. As a result, caution must be taken when relating relative amplitudes to relative conductivity between separate discrete targets.

In our formulation of the inverse problem, we perform a potential-field-style inversion on a single time channel, which reduces the ability of the inversion to constrain the depth of the causative features (i.e., loss of time-depth relationships). We alleviate this problem by using potential-field-style depth weighting, which is proportional to the spatial decay of the forward operator kernel. In our experience, if the regularization parameters are carefully chosen (L-curve analysis), the inverted anomaly depths match the depth of the actual causative features. This problem could also be alleviated by incorporating a reliable starting model into the inverse problem, as suggested by Schaa and Fullagar (2010).

Future work aims to investigate inverting multiple time channels either simultaneously or iteratively and using the differences in the location and amplitude of the predicted current system to estimate the conductivity and the conductivity structure of the subsurface. Furthermore, the method can be extended to airborne data (or any multitransmitter data) by incorporating primary-field coupling information and to borehole data by modifying the depth weighting to weighting based on the distance between cells and the observation point ( $\mathrm{Li}$ and Oldenburg, 2000). Finally, further research is being done into constraining the electric dipole inversion to form a consistent closed loop zero-divergence solution.

\section{CONCLUSION}

Under the quasistatic assumption, the magnetic field measured in the air at any given fixed time is a potential field and is determined by the subsurface current system. Conversely, we can invert measured magnetic fields (at a given fixed time) to determine the causative subsurface current system. In our formulation, we approximate these currents with a grid of 3D magnetic (closed loop current) or electric (line current) dipoles, which are solved for with a potentialfield style smooth-model inversion. Currently, the methodology has only been tested on relatively discrete (thin-sheet) bodies within a resistive medium (i.e., negligible background response), but future plans include investigating the potential to generalize the method to allow for an arbitrary background response. Synthetic work using plate models reveals that electric and magnetic dipoles (magnitude and vector orientation) can reveal significant information about the subsurface geology. Specifically, magnetic dipoles tend to con- centrate near the center of targets, whereas electric dipoles align themselves along the shallowest edge of the target. Orientation information can be estimated from the vector orientation of the dipole moments and/or from the locations of the peak magnetic and electric dipoles.

A field example over a deep mineral exploration target confirmed the conclusions drawn from the synthetic examples and the interpreted results (southwest shallow-dipping target at a depth of approximately $400 \mathrm{~m}$ ) were consistent with previous interpretations and drilling. A second example over a near-surface mine tailing highlighted the strength of being able to invert data using either magnetic or electric dipoles. Although the magnetic and electric dipole models were able to fit the data, the geologic interpretation using the electric dipole model was simpler and was interpreted to be more consistent with the believed geology.

Because the developed inversions can be run in a few seconds even for large subsurface grids, the magnetic and electric dipole models can be used and interpreted. This fast, approximate 3D inversion can be used as a starting point for more rigorous interpretation and/or, in some cases, as a stand-alone interpretation tool.

\section{ACKNOWLEDGMENTS}

We are thankful to Sudbury Integrated Nickel Operations, a Glencore Company, particularly W. Hughes, for the Joe Lake data and permission to publish the results. We are also grateful to Vale for permission to publish the data and results from the tailings pond example. Moreover, we are grateful to the following for financial support of this research: NSERC; Vale; Sudbury Integrated Nickel Operations, a Glencore Company; Wallbridge Mining; KGHM International; and the Centre for Excellence in Mining Innovation. M. Kolaj is grateful for an NSERC Alexander Graham Bell scholarship and an SEG George V. Keller Scholarship.

\section{REFERENCES}

Beran, L., B. Zelt, L. Pasion, S. Billings, K. Kingdon, N. Lhomme, L. Song, and D. Oldenburg, 2013, Practical strategies for classification of unexploded ordnance: Geophysics, 78, no. 1, E41-E46, doi: 10.1190/ geo2012-0236.1.

Christensen, N. B., 2002, A generic 1-D imaging method for transient electromagnetic data: Geophysics, 67, 438-447, doi: 10.1190/1.1468603.

Cox, L. H., G. A. Wilson, and M. S. Zhdanov, 2010, 3D inversion of airborne electromagnetic data using a moving footprint: Exploration Geophysics, 41, 250-259, doi: 10.1071/EG10003.

Fullagar, P. K., G. A. Pears, J. E. Reid, and R. Schaa, 2015, Rapid approximate inversion of airborne TEM: Exploration Geophysics, 46, 112-117, doi: $10.1071 / \mathrm{EG} 14046$.

Grant, F. S., and G. F. West, 1965, Interpretation theory in applied geophysics: McGraw-Hill.

Haber, E., D. W. Oldenburg, and R. Shekhtman, 2007, Inversion of time domain three-dimensional electromagnetic data: Geophysical Journal International, 171, 550-564, doi: 10.1111/j.1365-246X.2007.03365.x.

Huang, H., and J. Rudd, 2008, Conductivity-depth imaging of helicopterborne TEM data based on a pseudolayer half-space model: Geophysics, 73, no. 3, F115-F120, doi: 10.1190/1.2904984.

King, A., and J. Macnae, 2001, Modeling of the EM inductive-limit surface currents: Geophysics, 66, 476-481, doi: 10.1190/1.1444938.

Kolaj, M., and R. S. Smith, 2013, Using spatial derivatives of electromagnetic data to map lateral conductance variations in thin sheet models: Applications over mine tailings ponds: Geophysics, 78, no. 5, E225E235, doi: 10.1190/geo2012-0457.1.

Kolaj, M., and R. S. Smith, 2014, Mapping lateral changes in conductance of a thin sheet using time-domain inductive electromagnetic data: Geophysics, 79, no. 1, E1-E10, doi: 10.1190/geo2013-0219.1.

Kolaj, M., and R. S. Smith, 2015, A multiple transmitter and receiver electromagnetic system for improved target detection: Geophysics, 80, no. 4 , E247-E255, doi: 10.1190/geo2014-0466.1. 
Li, Y., and D. W. Oldenburg, 1996, 3-D inversion of magnetic data: Geophysics, 61, 394-408, doi: 10.1190/1.1443968.

Li, Y., and D. W. Oldenburg, 2000, Joint inversion of surface and three-component borehole magnetic data: Geophysics, 65, 540-552, doi: 10.1190/1 .1444749 .

Liu, G., and M. Asten, 1993, Conductance-depth imaging of airborne TEM data: Exploration Geophysics, 24, 655-662, doi: 10.1071/ EG993655.

Macnae, J., R. S. Smith, B. Polzer, Y. Lamontagne, and P. Klinkert, 1991, Conductivity-depth imaging of airborne electromagnetic step-response data: Geophysics, 56, 102-114, doi: 10.1190/1.1442945.

Macnae, J. C., and Y. Lamontagne, 1987, Imaging quasi-layered conductive structures by simple processing of transient electromagnetic data: Geophysics, 52, 545-554, doi: 10.1190/1.1442323.

Murray, R., C. Alvarez, and R. W. Groom, 1999, Modeling of complex electromagnetic targets using advanced non-linear approximator techniques: 69th Annual International Meeting, SEG, Expanded Abstracts, 271-274.

Nabighian, M. N., 1979, Quasi-static transient response of a conductive halfspace - An approximate representation: Geophysics, 44, 1700-1705, doi: 10.1190/1.1440931.

Nabighian, M. N., and J. C. Macnae, 1991, Time domain electromagnetic prospecting methods, in M. N. Nabighian, ed., Electromagnetic methods in applied geophysics: Part A and B: Applications: SEG, 427-520.

Oldenburg, D. W., E. Haber, and R. Shekhtman, 2013, Three dimensional inversion of multisource time domain electromagnetic data: Geophysics, 78, no. 1, E47-E57, doi: 10.1190/geo2012-0131.1.

Pasion, L., and D. Oldenburg, 2001, A discrimination algorithm for UXO using time domain electromagnetics: Journal of Environmental and Engineering Geophysics, 6, 91-102, doi: 10.4133/JEEG6.2.91.

Sattel, D., 1998, Conductivity information in three dimensions: Exploration Geophysics, 29, 157-162, doi: 10.1071/EG998157.

Sattel, D., 2004, The resolution of shallow horizontal structure with airborne EM: Exploration Geophysics, 35, 208-216.
Sattel, D., and J. Reid, 2006, Modeling of airborne EM anomalies with magnetic and electric dipoles buried in a layered earth: Exploration Geophysics, 37, 254-260, doi: 10.1071/EG06254.

Schaa, R., and P. K. Fullagar, 2010, Rapid approximate 3D inversion of transient electromagnetic (TEM) data: 80th Annual International Meeting, SEG, Expanded Abstracts, 650-654.

Smith, R. S., 2000, The realizable resistive limit: A new concept for mapping geological features spanning a broad range of conductances: Geophysics, 65, 1124-1127, doi: $10.1190 / 1.1444805$.

Smith, R. S., R. N. Edwards, and G. Buselli, 1994, An automatic technique for presentation of coincident-loop, impulse-response, transient, electromagnetic data: Geophysics, 59, 1542-1550, doi: 10.1190/1.1443543.

Smith, R. S., and T. J. Lee, 2001, The impulse response moments of a conductive sphere in a uniform field: A versatile and efficient electromagnetic model: Exploration Geophysics, 32, 113-118, doi: 10.1071/EG01113.

Smith, R. S., and T. J. Lee, 2002, The moments of the impulse response: A new paradigm for the interpretation of transient electromagnetic data: Geophysics, 67, 1095-1103, doi: 10.1190/1.1500370.

Smith, R. S., and A. S. Salem, 2007, A discrete conductor transformation of airborne electromagnetic data: Near Surface Geophysics, 5, 87-95.

Walker, P., and G. F. West, 1991, A robust integral equation solution for electromagnetic scattering by a thin plate in conductive media: Geophysics, 56, 1140-1152, doi: 10.1190/1.1443133.

Ward, S. H., and G. W. Hohmann, 1988, Electromagnetic theory for geophysical applications, in M. N. Nabighian, ed., Electromagnetic methods in applied geophysics: Theory: SEG, $130-311$.

Watts, A., 1997, Exploring for nickel in the 90's, or 'til depth us do part', in A. G. Gubins, ed., Proceedings of Exploration 97: Fourth Decennial International Conference on Mineral Exploration, 1003-1014.

West, G. F., J. C. Macnae, and Y. Lamontagne, 1984, A time-domain EM system measuring the step response of the ground: Geophysics, 49, 10101026, doi: 10.1190/1.1441716.

Zhdanov, M. S., 2002, Geophysical inverse theory and regularization problems: Elsevier Science. 